\title{
A cooperative spectrum sensing method based on information geometry and fuzzy c-means clustering algorithm
}

\author{
Shunchao Zhang ${ }^{1}$, Yonghua Wang ${ }^{1,2^{*}}$, Jiangfan $\mathrm{Li}^{1}$, Pin Wan ${ }^{1,3}$, Yongwei Zhang ${ }^{1}$ and $\mathrm{Nan}^{\mathrm{Li}}{ }^{1}$
}

\begin{abstract}
To improve spectrum sensing performance, a cooperative spectrum sensing method based on information geometry and fuzzy c-means clustering algorithm is proposed in this paper. In the process of signal feature extraction, a feature extraction method combining decomposition, recombination, and information geometry is proposed. First, to improve the spectrum sensing performance when the number of cooperative secondary users is small, the signals collected by the secondary users are split and reorganized, thereby logically increasing the number of cooperative secondary users. Then, in order to visually analyze the signal detection problem, the information geometry theory is used to map the split and recombine signals onto the manifold, thereby transforming the signal detection problem into a geometric problem. Further, use geometric tools to extract the corresponding statistical characteristics of the signal. Finally, according to the extracted features, the appropriate classifier is trained by the fuzzy c-means clustering algorithm and used for spectrum sensing, thus avoiding complex threshold derivation. In the simulation results and performance analysis section, the experimental results were further analyzed, and the results show that the proposed method can effectively improve the spectrum sensing performance.
\end{abstract}

Keywords: Cooperative spectrum sensing, Information geometry, Decomposition and recombination, Fuzzy c-means clustering algorithm

\section{Introduction}

With the development of wireless communication, spectrum resources have become increasingly scarce, but most of the existing spectrum resources have not been fully utilized. Cognitive radio (CR) technology allows secondary users (SUs) to access the spectrum when the authorized primary user (PU) is idle, thus effectively alleviating the spectrum scarcity problem [1]. In CR, spectrum sensing is a key step that is mainly used to sense the existence of the PU [2].

\subsection{Related work}

Classical spectrum sensing methods include energy detection, matched filter detection, and cyclic eigenvalue detec-

\footnotetext{
*Correspondence: sjzwyh@163.com

'School of Automation, Guangdong University of Technology, Guangzhou 510006, China

${ }^{2}$ State Key Laboratory of Management and Control for Complex Systems, Institute of Automation, Chinese Academy of Sciences, Beijing 100190, China Full list of author information is available at the end of the article
}

tion [3]. As the simplest spectrum sensing algorithm, energy detection has low computational complexity and does not require prior information from the PU. Therefore, this method has been widely used. However, the algorithm is susceptible to noise uncertainty, which will greatly reduce detection performance $[4,5]$. Matching filter detection is the optimal signal detection algorithm when all the information of the PU is known. However, the disadvantages of this method are also very obvious, because it requires prior knowledge of the PU that include the packet format and sequence, and the modulation type $[6,7]$. The calculation of cyclic eigenvalue detection is relatively complicated, so it cannot be detected in real time; therefore, rapid detection is not possible [8].

The application of random matrix theory to spectrum sensing has attracted the interest of many researchers $[9,10]$. By acquiring the sensing data of multiple SUs and composing the sampling signal matrix, and then calculating the covariance matrix, the corresponding eigenvalue is finally calculated as the decision statistic. Nowadays, 
many cooperative spectrum sensing algorithms based on random matrices have been proposed. Liu et al. proposed a maximum to minimum eigenvalue (MME) spectrum sensing method. The modified method uses the extracted MME feature value as a statistical feature of the signal and compares with a preset threshold to determine whether the PU exists [11]. However, when the number of sampling points is insufficient, the detection performance is obviously degraded. Liu et al. proposed a spectrum sensing method for the difference between the maximum eigenvalue and the average energy (DMEAE) . The method uses the DMEAE feature as a statistical feature of the signal and then compares it with a preset threshold to achieve spectrum sensing [12]. Tulino et al. proposed a spectrum sensing method based on the difference between the maximum eigenvalue and the minimum feature (DMM) [13]. Similar to the above method, the DMM feature is also used as a statistical feature, and spectrum sensing is implemented by comparing with a preset threshold. However, this method has poor perceived performance when the number of cooperative SUs is small and the signal-to-noise ratio (SNR) is low. A statistical feature extraction method based on decomposition and recombination (DAR) is proposed. To logically increase the number of cooperative users, the method firstly splits and reorganizes the signal matrix, thereby effectively improving the spectrum sensing performance [14]. From the above analysis, it can be known that the traditional spectrum sensing based on random matrix needs to derive and calculate the threshold of the decision in advance. The whole process is complex, and there are problems such as inaccurate thresholds.

With the rapid development of information geometry theory, the concept of statistical manifolds is used to transform signal detection problems into geometric problems on manifolds, and then geometric tools can be used to visually analyze detection problems. Liu et al. used information geometry theory to detect radar signals. At the same time, a matrix constant false alarm rata (CFAR) and a distance detector based on geodesic were proposed [15]. Chen applied the information geometry method to spectrum sensing, increased the measurement of manifolds, and obtained the decision threshold through simulation [16]. Lu et al. used the matching method to obtain the closed expression of the decision threshold, which has higher computational complexity [17]. However, in spectrum sensing, the derivation of the threshold is not only complicated, but there is always some deviation in using the fixed decision threshold to determine whether the PU exists.

In recent years, machine learning has developed rapidly, which also provides a new idea for spectrum sensing. Spectrum sensing can be considered as a problem of two classifications that is whether the PU exists [18-20].
Kumar proposed a spectrum sensing method based on $\mathrm{K}$-means clustering algorithm and energy feature. The method takes the energy value of the signal as the feature and then uses K-means clustering algorithm to classify these features [21]. Zhang et al. proposed a spectrum sensing method based on K-means and signal features that combines the feature extraction methods in the random matrix and selects MME, DMM, and DMEAE as the characteristics of training and classification [22]. Thilina et al. used K-means, Gaussian mixture model (GMM) in unsupervised learning, and neural network (NN) and support vector machine (SVM) in supervised learning to study spectrum perception [23]. Xue et al. proposed a cooperative spectrum sensing algorithm based on unsupervised learning. The dominant features and the maximum and minimum eigenvalues are used as features. K-means clustering and GMM are selected as the learning framework [24]. Compared with the traditional spectrum sensing method, spectrum sensing based on machine learning can effectively eliminate the cumbersome threshold calculation and has better adaptability. Similarly, the method does not need to know the priori information of the PU.

\subsection{Contributions}

Based on the above researches, this paper proposes a cooperative spectrum sensing method based on information geometry and fuzzy c-means (FCM) clustering algorithm (IGFCM). In the feature extraction process, the order-DAR (O-DAR) and interval-DAR (I-DAR) are introduced to obtain two new matrices, then two covariance matrices of two new matrices are calculated separately, and then two covariance matrices are mapped to the manifold using information geometry theory. Then, use the geodesic distance to calculate the distance on the manifold and use it as a feature. Finally, the FCM clustering algorithm is used to implement spectrum sensing. The spectrum sensing method proposed in this paper does not require any prior information about the communication system, and the unmarked training data is more easily obtained. In the experiment, the spectrum sensing performance of IGFCM was further analyzed. The simulation results show that the method effectively improves the spectrum sensing performance.

\subsection{Methods or experimental}

The main structure and arrangement of this paper are as follows. Section 2 mainly introduces the system model of cooperative spectrum sensing. Section 3 is to improve the spectrum sensing performance in the case of a small number of cooperative users and to analyze the spectrum sensing problem more intuitively. A signal feature extraction method based on split recombination and information geometry is proposed. Section 4 uses FCM clustering algorithm to achieve spectrum sensing. Section 5 uses 
the amplitude modulation signal to verify the proposed method.

\section{Cooperative spectrum sensing system model}

According to the perception of PU by a single SU in a cognitive radio network (CRN), the following binary hypothesis [25] about PU can be obtained. $H_{0}$ indicates that the PU signal does not exist, and $H_{1}$ indicates that the PU signal exists.

$$
x(n)= \begin{cases}w(n) & H_{0} \\ s(n)+w(n) & H_{1}\end{cases}
$$

where $s(n)$ represents the signal transmitted by the PU and $w(n)$ is the ambient noise. Since CR is primarily used for relatively fixed networks, the actual channel model is similar to additive white Gaussian noise (AWGN). The systems false alarm probability $\left(P_{f}\right)$ and detection probability $\left(P_{d}\right)$ can be defined as:

$$
\begin{aligned}
& P_{f}=P\left[H_{1} \mid H_{0}\right] \\
& P_{d}=P\left[H_{1} \mid H_{1}\right]
\end{aligned}
$$

In CRN, spectrum sensing is often done in complex environments. Therefore, the SU needs to consider peripheral multi-path fading, shadow effects, and hidden terminals in the process of sensing the PU [26, 27]. Cooperative spectrum sensing reduces the impact of environmental factors by increasing the diversity of SUs. Therefore, to improve the performance of the spectrum sensing system, the method of multi-SU cooperative spectrum sensing is adopted. First, SUs collect information about the authorized channel and then transmits the information to a fusion center (FC) through the reporting channel; finally, the unified processing by the FC and the final decision is made. Cooperative spectrum sensing system model is shown in Fig. 1.

Assuming that there are $M$ SUs in a CRN, the signals collected by the $M$ SUs can form a signal vector matrix $x_{i}=\left[x_{i}(1), x_{i}(2), \ldots, x_{i}(N)\right]$, where $\mathbf{X}=\left[x_{1}, x_{2}, \ldots, x_{M}\right]^{T}$ represents the signal sample value of the $i$ th SU. Therefore, $\mathrm{X}$ is a matrix of $M \times N$ dimensions.

$$
\mathbf{X}=\left[x_{1}, x_{2}, \ldots, x_{M}\right]^{T}=\left[\begin{array}{cccc}
x_{1}(1) & x_{1}(2) & \cdots & x_{1}(N) \\
x_{2}(1) & x_{2}(2) & \cdots & x_{2}(N) \\
\vdots & \vdots & \ddots & \vdots \\
x_{M}(1) & x_{M}(2) & \cdots & x_{M}(N)
\end{array}\right]
$$

For ease of reference, the symbols and notations used in this paper are summarized in Table 1.

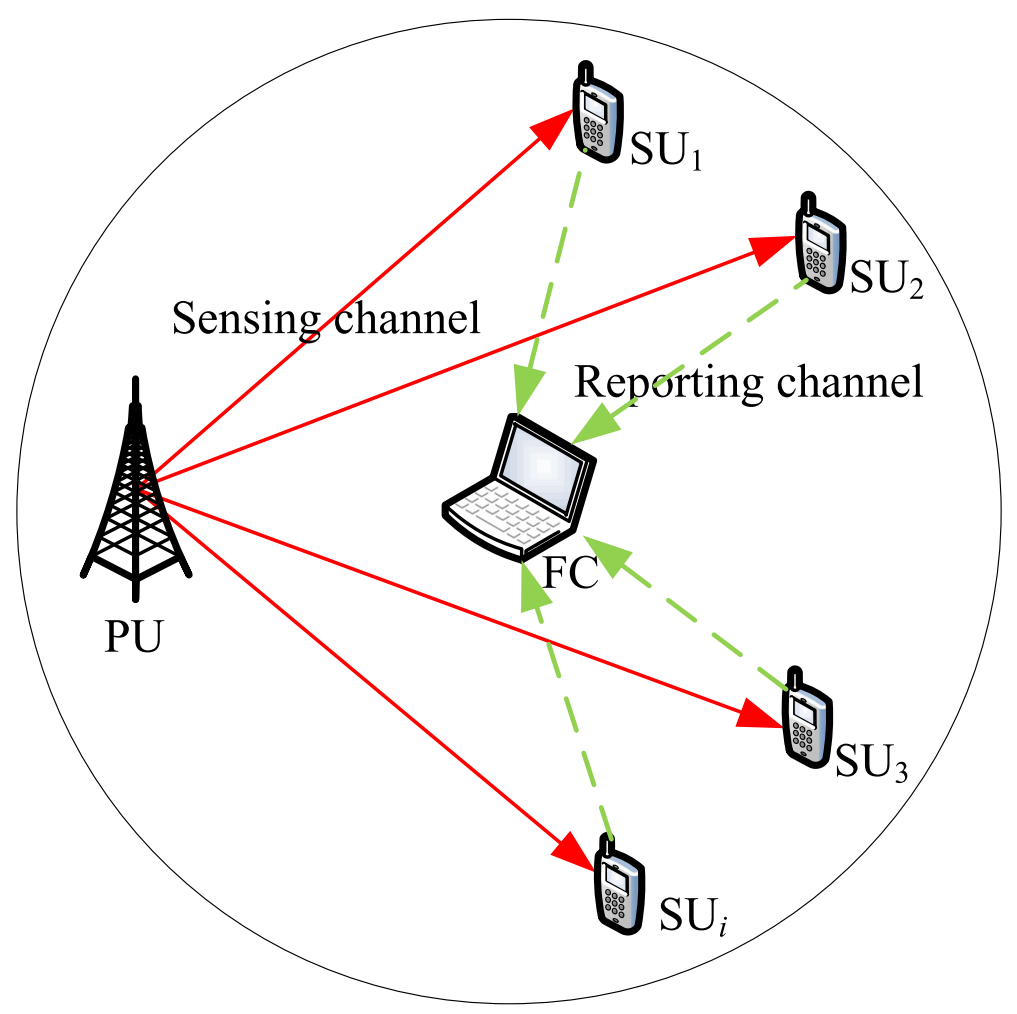

Fig. 1 Cooperative spectrum sensing system model 
Table 1 Summary symbols and notations

\begin{tabular}{|c|c|}
\hline Symbols & Notations \\
\hline$w(n)$ & Gaussian noise \\
\hline$s(n)$ & The signal transmitted by the PU \\
\hline$x(n)$ & The signal received by the SU \\
\hline N & The number of sampling points \\
\hline M & The number of SUs participating in CSS \\
\hline$H_{1}, H_{0}$ & PU exists, PU does not exist \\
\hline$p_{f}, p_{d}$ & False alarm probability and detection probability \\
\hline$x_{i}$ & The signal acquired by the ith SU \\
\hline $\mathbf{x}$ & Signal matrix \\
\hline $\mathbf{R}$ & Covariance matrix corresponding to $\mathbf{X}$ \\
\hline $\mathbf{X}^{T}$ & The transposition of $\mathbf{X}$ \\
\hline $\mathbf{Y}_{O-D A R}, \mathbf{Y}_{\text {l-DAR }}$ & A matrix after O-DAR and I-DAR \\
\hline \multirow[t]{2}{*}{$\mathbf{R}^{O}, \mathbf{R}^{\prime}$} & Covariance matrix corresponding to $\mathbf{Y}_{O-D A R}$ \\
\hline & and $\mathbf{Y}_{l-D A R}$ \\
\hline$x$ & An n-dimensional sample \\
\hline$\theta$ & A parameter vector, a point on the manifold \\
\hline$\Omega$ & A random variable \\
\hline S & The probability distribution function family \\
\hline$\Theta$ & Probability distribution space \\
\hline \multirow[t]{2}{*}{$\mathbf{R}_{w}, \mathbf{R}_{s}+\mathbf{R}_{w}$} & Covariance matrix corresponding to $\mathbf{X}$ under \\
\hline & $H_{0}$ and $H_{1}$ \\
\hline 9 & The split parameter \\
\hline s & The length of the split signal vector after splitting \\
\hline \multirow{2}{*}{$\mathbf{R}_{k}^{O}, \mathbf{R}_{k}^{\prime}$} & The $k$ th noise signal covariance matrix after \\
\hline & $\begin{array}{l}\text { O-DAR and I-DAR } \\
\text { Iteration step size }\end{array}$ \\
\hline & Iteration step \\
\hline$\overline{\mathbf{R}}^{\bigcirc}, \overline{\mathbf{R}}^{\prime}$ & Riemann mean of $\mathbf{R}_{k}^{O}$ and $\mathbf{R}_{k}^{\prime}$ \\
\hline \multirow[t]{2}{*}{$d_{1}, d_{2}$} & Distance between the perceived signal and \\
\hline & reference point $\overline{\mathbf{R}}^{\bigcirc}$ and $\overline{\mathbf{R}}^{\prime}$ on the manifold \\
\hline D & Geodesic distance feature vector (GDFV) \\
\hline$\overline{\mathrm{D}}$ & Training set \\
\hline \multirow[t]{2}{*}{$Z_{c}$} & The set of training feature vectors belonging \\
\hline & to class C \\
\hline$\Psi_{c}$ & Center point of $Z_{c}$ \\
\hline$\underline{\underline{u_{c j}}}$ & The membership degree \\
\hline \multirow[t]{2}{*}{$\overline{\overline{\mathbf{D}}}$} & The feature extracted under the channel of the \\
\hline & unknown PU state \\
\hline C & Number of clusters \\
\hline$v$ & Smoothing index or fuzzy weighted index \\
\hline$m$ & Error metric \\
\hline$\underline{\varepsilon}$ & Fault tolerance factor \\
\hline
\end{tabular}

3 Feature extraction based on decomposition and recombination and information geometry

\subsection{Feature extraction model}

In the feature extraction process, the noise environment needs to be estimated first, and two covariance matrices are obtained by splitting and recombining the noise signal matrix in sequence and interval. The model of feature extraction based on decomposition and recombination and information geometry is shown in Fig. 2. In order to accurately estimate the noise environment, collect enough noise signal matrices and perform O-DAR and I-DAR and covariance transformation (as shown in the box in Fig. 2). Then, use the Riemann mean calculation method to solve the Riemann mean of these covariance matrices. Similarly, the signal matrix with the perception is also subjected to two kinds of split recombination, and the covariance matrix is transformed. Finally, the distance from the covariance matrix obtained from the environment to be perceived to the Riemann mean is calculated. Then, use this distance as a statistical feature of the signal.

\subsection{Information geometry overview}

According to the matrix $\mathbf{X}$, the corresponding covariance matrix can be calculated as shown in Eq. 5 .

$$
\mathbf{R}=\frac{1}{N} \mathbf{X X}^{T}
$$

From the theory of information geometry, we assume a set of probability density functions $p(x \mid \theta)$, where $x$ is an $n$-dimensional sample belonging to the random variable $\Omega, x \in \Omega \in C^{n} . \theta$ is an $m$-dimensional parameter vector, $\theta \in \Theta \subseteq C^{m}$. Therefore, the probability distribution space can be described by parameter set $\Theta$. The probability distribution function family $S$ is as shown in Eq. 6 .

$$
S=\left\{p(x \mid \theta) \mid \theta \in \Theta \subseteq C^{m}\right\}
$$

Under a certain topological structure, $S$ can form a microscopic manifold, called a statistical manifold, and $\theta$ is the

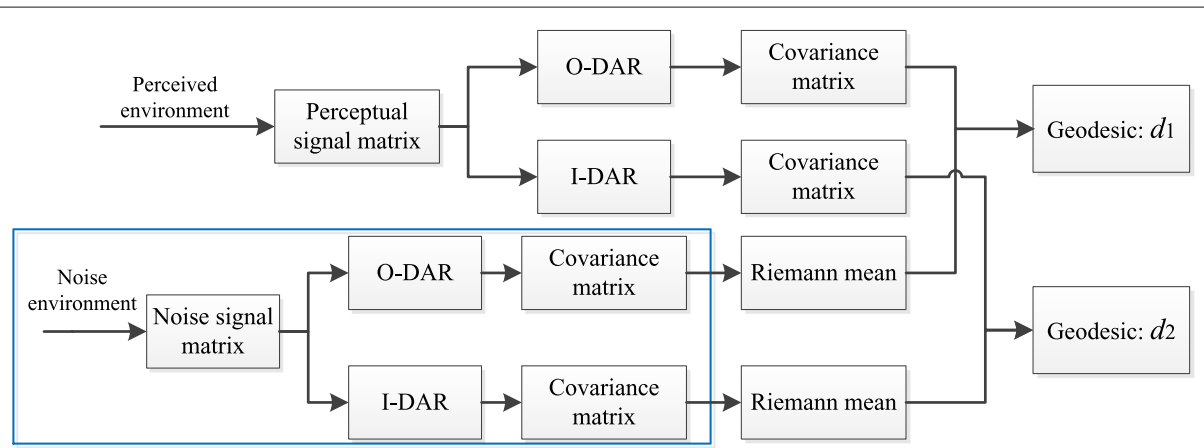

Fig. 2 Feature extraction model 
coordinate of the manifold. From the perspective of information geometry, the probability density function can be parameterized by the corresponding covariance matrix. Under the two hypotheses $H_{0}$ and $H_{1}$ of spectrum sensing, the signal can be mapped to a point that is $\mathbf{R}_{w}$ or $\mathbf{R}_{s}+\mathbf{R}_{w}$, on the manifold. $\mathbf{R}_{w}$ and $\mathbf{R}_{s}+\mathbf{R}_{w}$ are respectively the covariance calculated from the noise matrix and the signal matrix. In particular, both $\mathbf{R}_{w}$ and $\mathbf{R}_{s}+\mathbf{R}_{w}$ are Toeplitz Hermitian positive definite matrices [17]. Therefore, a symmetric positive definite (SPD) matrix space composed of a covariance matrix can be defined as an SPD manifold.

\subsection{Decomposition and recombination}

In this section, we first split and reorganize the signal matrix for SU to logically increase the number of cooperative SUs. The DAR is divided into O-DAR and I-DAR. At the same time, the O-DAR and I-DAR are used to process the signal vector perceived by the SU. The specific algorithm is as follows [14]:

In the process of O-DAR, $x_{i}$ will be sequentially split into sub-signal vectors of $q(q>0)$ segment $s=N / q$ long. Then, the result of splitting $x_{i}$ is as follows:

$$
x_{i}\left\{\begin{array}{l}
x_{i 1}=\left[x_{i}(1), x_{i}(2), \ldots, x_{i}(s)\right] \\
x_{i 2}=\left[x_{i}(s+1), x_{i}(s+2), \ldots, x_{i}(2 s)\right] \\
\vdots \\
x_{i q}=\left[x_{i}((q-1) s+1), x_{i}((q-1) s+2), \ldots, x_{i}(q s)\right]
\end{array}\right.
$$

The signal vector in Eq. 4 is split according to Eq. 7, and then, the split sub-signal vector is recombined to obtain a $q M \times s$ dimensional signal matrix $\mathbf{Y}_{O-D A R}$.

$\mathbf{Y}_{O-D A R}=\left[\begin{array}{l}x_{11} \\ \vdots \\ x_{1 q} \\ \vdots \\ x_{i m} \\ \vdots \\ x_{M q}\end{array}\right]=\left[\begin{array}{cccc}x_{1}(1) & x_{1}(2) & \cdots & x_{1}(s) \\ \vdots & & & \\ x_{1}((q-1) s+1) & x_{1}((q-1) s+2) & \cdots & x_{1}(q s) \\ \vdots & & & \\ x_{i}((m-1) s+1) & x_{i}((m-1) s+2) & \cdots & x_{i}(m s) \\ \vdots & & & \\ x_{M}((q-1) s+1) & x_{M}((q-1) s+2) & \cdots & x_{M}(q s)\end{array}\right]$

In the process of I-DAR, select sampling points in the sampled data every $q-1$ units and then recombine the signal matrix $\mathbf{X}$. The sampled data is separated by $q-1$ units, the sample points are reselected, and the signal matrix is recombined. According to I-DAR, the sampled data can be split into sub-signal vectors of $q(q>0)$ segment $s=N / q$ long. Then, the result of splitting $x_{i}$ is as follows:

$$
x_{i}\left\{\begin{array}{l}
x_{i 1}=\left[x_{i}(1), x_{i}(q+1), \ldots, x_{i}((s-1) q+1)\right] \\
x_{i 2}=\left[x_{i}(2), x_{i}(q+2), \ldots, x_{i}((s-1) q+2)\right] \\
\vdots \\
x_{i q}=\left[x_{i}(q), x_{i}(q+q), \ldots, x_{i}((s-1) q+q)\right]
\end{array}\right.
$$

The signal vector in Eq. 4 is split according to Eq. 9, and then, the split sub-signal vector is recombined to obtain a $q M \times s$ dimensional signal matrix $\mathbf{Y}_{I-D A R}$.

$$
\mathbf{Y}_{I-D A R}=\left[\begin{array}{l}
x_{11} \\
\vdots \\
x_{1 q} \\
\vdots \\
x_{i m} \\
\vdots \\
x_{M q}
\end{array}\right]=\left[\begin{array}{llll}
x_{1}(1) & x_{1}(q+1) & \cdots & x_{1}((s-1) q+1) \\
\vdots & & & \\
x_{1}(q) & x_{1}(q+q) & \cdots & x_{1}((s-1) q+q) \\
\vdots & & & \\
x_{i}(m) & x_{i}(q+m) & \cdots & x_{i}((s-1) q+m) \\
\vdots & & & \\
x_{M}(q) & x_{M}(q+q) & \cdots & x_{M}((s-1) q+q)
\end{array}\right]
$$

According to $\mathbf{Y}_{O-D A R}$ and $\mathbf{Y}_{I-D A R}$, the corresponding covariance matrices $\mathbf{R}^{O}$ and $\mathbf{R}^{I}$ can be calculated.

$$
\begin{aligned}
\mathbf{R}^{O} & =\frac{1}{s} \mathbf{Y}_{O-D A R} \mathbf{Y}_{O-D A R}{ }^{T} \\
\mathbf{R}^{I} & =\frac{1}{s} \mathbf{Y}_{I-D A R} \mathbf{Y}_{I-D A R}{ }^{T}
\end{aligned}
$$

\subsection{Riemann mean}

First, SUs collect $P$ environmental noise matrices. These noise matrices are then processed using O-DAR and IDAR, and the covariance matrix will be calculated. Thus, we can obtain $\mathbf{R}_{k}^{O}(k=1,2, \ldots, P)$ and $\mathbf{R}_{k}^{I}(k=1,2, \ldots, P)$ matrices. Their Riemann mean objective functions are shown in Eqs. 13 and 14, respectively.

$$
\begin{gathered}
\Phi\left(\overline{\mathbf{R}}^{O}\right)=\frac{1}{P} \sum_{k=1}^{P} \mathrm{D}\left(\mathbf{R}_{k}^{O}, \overline{\mathbf{R}}^{O}\right) \\
\Phi\left(\overline{\mathbf{R}}^{I}\right)=\frac{1}{P} \sum_{k=1}^{P} \mathrm{D}\left(\mathbf{R}_{k}^{I}, \overline{\mathbf{R}}^{I}\right)
\end{gathered}
$$

$\overline{\mathbf{R}}^{O}$ and $\overline{\mathbf{R}}^{I}$ are the matrix when $\Phi(\bullet)$ takes the minimum value, where $\mathrm{D}(\bullet, \bullet)$ is the geodesic distance of two points on the manifold described below.

$$
\begin{gathered}
\overline{\mathbf{R}}^{O}=\arg \min \Phi\left(\overline{\mathbf{R}}^{O}\right) \\
\overline{\mathbf{R}}^{I}=\arg \min \Phi\left(\overline{\mathbf{R}}^{I}\right)
\end{gathered}
$$


Assume that for the case where there are two points $\mathbf{R}_{1}$ and $\mathbf{R}_{2}$ on the matrix manifold, $\overline{\mathbf{R}}$ is located at the midpoint of the geodesic line connecting the two points $\mathbf{R}_{1}$ and $\mathbf{R}_{2}$ on the manifold. Its expression is as shown in Eq. 17.

$$
\overline{\mathbf{R}}=\mathbf{R}_{1}^{1 / 2}\left(\mathbf{R}_{1}^{-1 / 2} \mathbf{R}_{2} \mathbf{R}_{1}^{-1 / 2}\right)^{1 / 2} \mathbf{R}_{2}^{1 / 2}
$$

If $P>2$, the Riemann mean will be difficult to calculate. Literatures $[28,29]$ give a method of iteratively calculating $\overline{\mathbf{R}}$ using the gradient descent algorithm, and finally obtain the Riemann mean calculation formula as shown in Eq. 18.

$$
\overline{\mathbf{R}}_{l+1}=\overline{\mathbf{R}}_{l}^{1 / 2} e^{-\frac{\tau}{P} \sum_{k=1}^{P} \log \left(\overline{\mathbf{R}}_{l}^{-1 / 2} \mathbf{R}_{k} \overline{\mathbf{R}}_{l}^{-1 / 2}\right)} \overline{\mathbf{R}}_{l}^{1 / 2}, \quad 0 \leq \tau \leq 1
$$

where $\tau$ is the step size of iteration and $l$ indicates the number of iteration steps. Therefore, we use the gradient descent algorithm to calculate the Riemann matrix, and get $\overline{\mathbf{R}}^{O}$ and $\overline{\mathbf{R}}^{I}$.

\subsection{Geodesic distance}

The study of a geometric structure is mainly to study some properties such as distance, tangent, and curvature on the structure. There are many ways to measure the distance between two probability distributions on a statistical manifold. The most common is the geodesic distance.

Assuming $\theta$ is a point on the manifold, the metric on the statistical manifold can be defined by $\mathrm{G}(\theta)$ of the following equation, called the Fisher information matrix.

$$
\mathrm{G}(\theta)=\mathrm{E}\left[\frac{\partial \ln p(x \mid \theta)}{\partial \theta_{i}} \cdot \frac{\partial \ln p(x \mid \theta)}{\partial \theta_{j}}\right]
$$

Due to the nature of the manifold curvature, we determine the distance between the two points by defining the length of the curve connecting the two points on the manifold. Consider an arbitrary curve $\theta(t)\left(t_{1} \leq t \leq t_{2}\right)$ between two points $\theta_{1}$ and $\theta_{2}$ on an arbitrary manifold, where $\theta\left(t_{1}\right)=$ $\theta_{1}, \theta\left(t_{2}\right)=\theta_{2}$. Then, the distance between $\theta_{1}$ and $\theta_{2}$ can be obtained along the curve $\theta(t)$ [30].

$$
\mathrm{D}\left(\theta_{1}, \theta_{2}\right) \triangleq \int_{t_{1}}^{t_{2}} \sqrt{\left(\frac{d \theta}{d t}\right)^{T} \mathrm{G}(\theta)\left(\frac{d \theta}{d t}\right) d t}
$$

It can be seen that the distance between $\theta_{1}$ and $\theta_{2}$ depends on the selection of the curve $\theta(t)$. We call the curve that makes Eq. 20 have the smallest distance as the geodesic, and call the corresponding distance as the geodesic distance.

For any probability distribution, the calculation of geodesic distance is more complicated, which has some adverse effects on its application. For a multivariate Gaussian distribution family with the same mean but different covariance matrices, consider the two members of $\mathbf{R}_{1}$ and $\mathbf{R}_{2}$ in the covariance matrix. The geodesic distance between them is shown in the following Eq. 21 [31].

$$
\begin{aligned}
\mathrm{D}\left(\mathbf{R}_{1}, \mathbf{R}_{2}\right) & \triangleq \sqrt{\frac{1}{2} \operatorname{tr} \log ^{2}\left(\mathbf{R}_{1}^{-1 / 2} \mathbf{R}_{2} \mathbf{R}_{1}^{-1 / 2}\right)} \\
& =\sqrt{\frac{1}{2} \sum_{i=1}^{n} \log ^{2} \eta_{i}}
\end{aligned}
$$

where $\eta_{i}$ is the $i$ eigenvalues of the matrix $\mathbf{R}_{1}^{-1 / 2} \mathbf{R}_{2} \mathbf{R}_{1}^{-1 / 2}$.

According to the feature extraction process and the above analysis, the signal matrix to be perceived is split and recombined in sequence and interval, and the covariance matrix is transformed to obtain $\mathbf{R}_{O}$ and $\mathbf{R}_{I}$. Then, we use Eq. 21 to solve the corresponding geodesic distance.

$$
\begin{aligned}
d_{1}=\mathrm{D}\left(\mathbf{R}^{O}, \overline{\mathbf{R}}^{O}\right) & \triangleq \sqrt{\frac{1}{2} \operatorname{trlog} \log ^{2}\left(\left(\mathbf{R}^{O}\right)^{-1 / 2} \overline{\mathbf{R}}^{O}\left(\mathbf{R}^{O}\right)^{-1 / 2}\right)} \\
& =\sqrt{\frac{1}{2} \sum_{i=1}^{q M} \log ^{2} \eta_{i}}
\end{aligned}
$$

$$
\begin{aligned}
d_{2} & =\mathrm{D}\left(\mathbf{R}^{I}, \overline{\mathbf{R}}^{I}\right) \triangleq \sqrt{\frac{1}{2} \operatorname{trlog} \log ^{2}\left(\left(\mathbf{R}^{I}\right)^{-1 / 2} \overline{\mathbf{R}}^{I}\left(\mathbf{R}^{I}\right)^{-1 / 2}\right)} \\
& =\sqrt{\frac{1}{2} \sum_{i=1}^{q M} \log ^{2} \eta_{i}}
\end{aligned}
$$

According to the geodesic $d_{1}$ and $d_{1}$, a two-dimensional feature vector $\mathbf{D}=\left[d_{1}, d_{2}\right]$ is used to represent the signal sensed by the SU. Finally, the feature vector $\mathbf{D}$ is used for spectrum sensing.

\section{Cooperative spectrum sensing based on FCM clustering algorithm}

The FCM clustering algorithm is based on the partitioning method to obtain the clustering result.The basic idea is to divide similar samples into the same class as much as possible. The FCM clustering algorithm is an improvement of the common K-means clustering algorithm. The common $\mathrm{K}$-means clustering algorithm is hard to divide the data, and the FCM clustering algorithm is a flexible fuzzy partitioning [32].Compared with traditional spectrum sensing methods, cooperative spectrum sensing based on FCM clustering algorithm not only eliminates complex threshold derivation but also has adaptability. The overall flow of the IGFCM method described in this paper is shown in Fig. 3. 


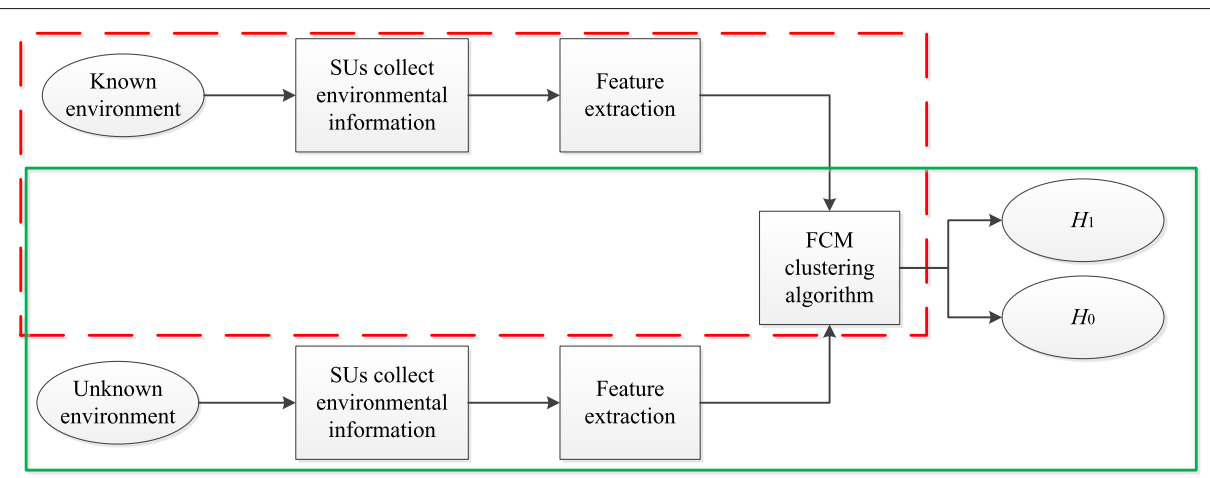

Fig. 3 The overall flow of the IGFCM method

The method of IGFCM is divided into two parts. In the first part, the red box indicates the training process. In the second part, the spectrum sensing process is represented in the green box.

\subsection{Training process based on FCM}

Before training, we need to prepare a training set $\overline{\mathrm{D}}$ :

$$
\overline{\mathrm{D}}=\left[\mathbf{D}_{1}, \mathbf{D}_{2}, \ldots, \mathbf{D}_{J}\right]
$$

Among them, $\mathbf{D}_{j}$ is the feature vector extracted in the third section and $J$ represents the number of training feature vectors. The clustering algorithm divides the unlabeled training feature vectors into $C$ non-overlapping clusters. Let $Z_{c}$ denote the set of training feature vectors belonging to class $c$, where $c=1,2, \ldots, C$, then

$$
Z_{c}=\left\{\mathbf{D}_{j} \mid \mathbf{D}_{j} \in \text { Cluster } c \forall j\right\}
$$

The class $Z_{c}$ has a corresponding center $\Psi_{c}$, and each sample $\mathbf{D}_{j}$ belongs to $\Psi_{c}$ with a membership degree of $u_{c j}$ and $0<u_{c j}<1$. The objective function $\Gamma$ of the FCM clustering algorithm is shown in Eq. 26, and the constraint condition is shown in Eq. 27.

$$
\begin{gathered}
\Gamma=\sum_{c=1}^{C} \sum_{j=1}^{J} u_{c j}^{m}\left\|\mathbf{D}_{j}-\Psi_{c}\right\|^{2} \\
\sum_{c=1}^{C} u_{c j}=1, \quad \forall j=1,2, \ldots, J
\end{gathered}
$$

where $\left\|\mathbf{D}_{j}-\Psi_{c}\right\|^{2}$ is an error metric and $m$ is a weighted power exponent of the membership degree $u_{c j}$, which may also be referred to as a smoothness index or a fuzzy weighted index, and $m>1$.

Using Lagrange to collate the objective function $\Gamma$ and constraints, the objective function shown in Eq. 28 is obtained.

$$
\begin{aligned}
\Gamma= & \sum_{c=1}^{C} \sum_{j=1}^{J} u_{c j}^{m}\left\|\mathbf{D}_{j}-\Psi_{c}\right\|^{2}+\lambda_{1}\left(\sum_{c=1}^{C} u_{c 1}-1\right) \\
& +\ldots+\lambda_{j}\left(\sum_{c=1}^{C} u_{c j}-1\right)+\ldots+\lambda_{J}\left(\sum_{c=1}^{C} u_{c J}-1\right)
\end{aligned}
$$

Then, the membership degree $u_{c j}$ and the cluster center $\Psi_{c}$ are respectively derived, and the constraint condition is substituted [33, 34], thereby obtaining the calculation formulas of $u_{c j}$ and $\Psi_{c}$, as shown in the Eqs. 29 and 30.

$$
\begin{gathered}
u_{c j}=\frac{1}{\sum_{k=1}^{C}\left(\frac{\left\|\mathbf{D}_{j}-\Psi_{c}\right\|}{\left\|\mathbf{D}_{j}-\Psi_{k}\right\|}\right)^{\frac{2}{m-1}}} \\
\Psi_{c}=\frac{\sum_{j=1}^{J}\left(\mathbf{D}_{j} u_{c j}^{m}\right)}{\sum_{j=1}^{J} u_{c j}^{m}}
\end{gathered}
$$

The training process based on the FCM clustering algorithm is as follows:

Step 1 Input training data set $\overline{\mathrm{D}}$, number of clusters $C$, smoothing index $m$, initialization membership $u_{c j}$, and fault tolerance factor $\varepsilon$

Step 2 Calculate the class center $\Psi_{c}$ by Eq. 30

Step 3 Calculate $v=\left\|\mathbf{D}_{j}-\Psi_{c}\right\|^{2}$, if $v<\varepsilon$, the algorithm stops; otherwise, continue to step 4

Step 4 Recalculate the membership degree $u_{c j}$ according to Eq. 29, return to step 2

Step 5 Output class center point $\Psi_{c}$

\subsection{Spectrum sensing process based on FCM}

After the training is successful, we can get a classifier for spectrum sensing, as shown in Eq. 31.

$$
\frac{\left\|\overline{\overline{\mathbf{D}}}-\Psi_{1}\right\|}{\min _{c=2,3, \ldots, C}\left\|\overline{\overline{\mathbf{D}}}-\Psi_{c}\right\|}>\xi
$$

In Eq. 31, $\overline{\overline{\mathbf{D}}}$ denotes an unknown perceptual signal feature vector. If Eq. 31 is satisfied, it indicates that the PU signal exists and the channel is not available; otherwise, the PU signal does not exist and the channel can be used. 


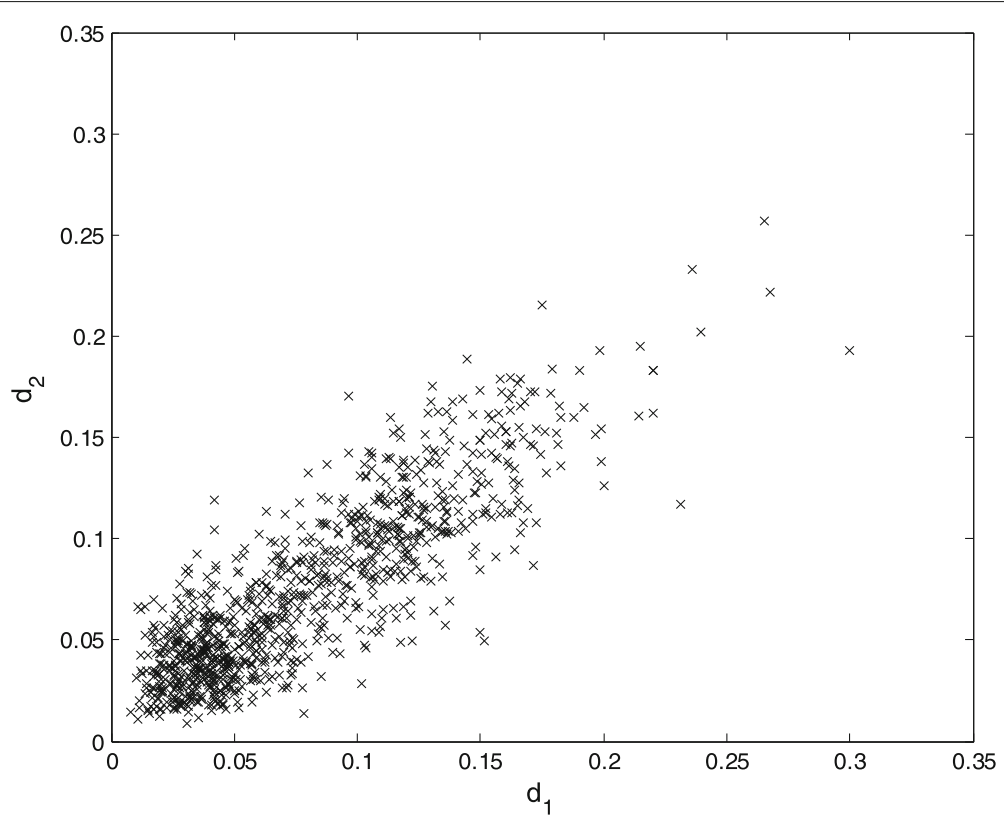

Fig. 4 Unclassified feature vectors

The parameter $\xi$ is used to control the probability of missed detection and false alarm probability in the sensing process [21].

\section{Simulation results and performance analysis}

The cooperative spectrum sensing algorithm based on fuzzy c-means clustering algorithm is simulated and analyzed in this section. The simulation PU signal is the amplitude modulation (AM) signal, and the noise is Gaussian white noise. In order to ensure the accuracy of the experiment, according to the feature extraction method described in Section 4, 2000 signal feature vectors were extracted, of which 1000 are training samples and 1000 are used as test samples.

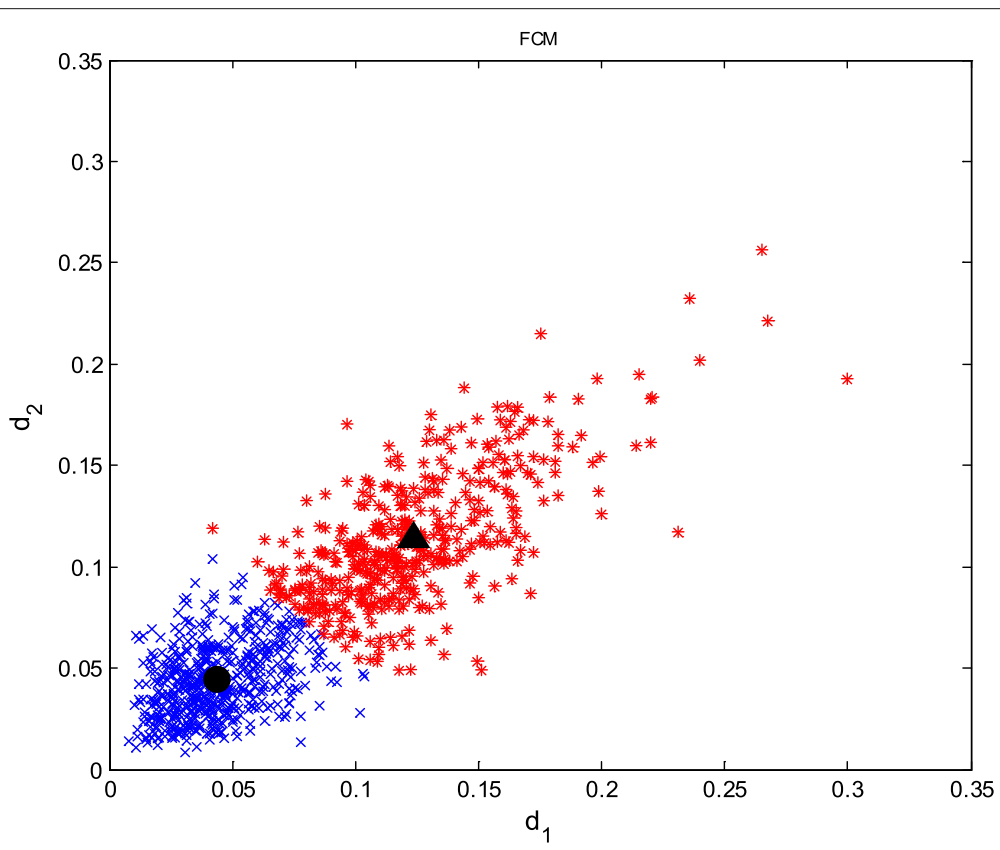

Fig. 5 Classified feature vectors 


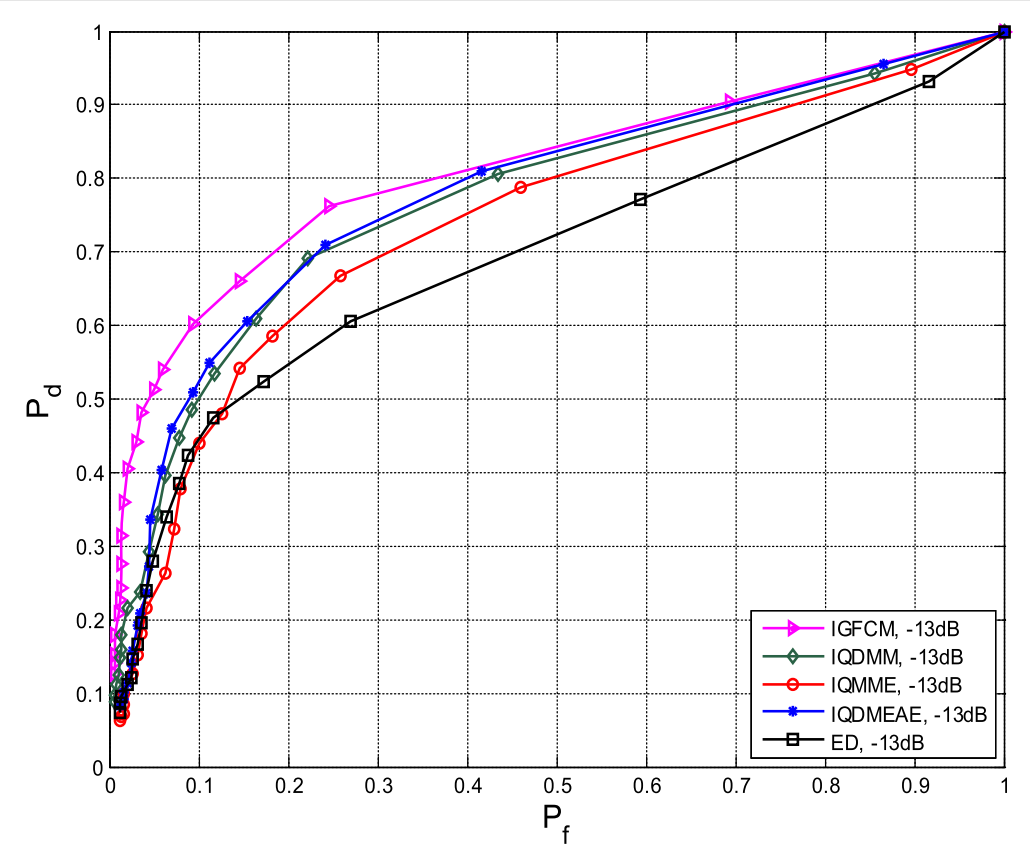

Fig. 6 ROC curves for different algorithms at $S N R=-13 \mathrm{~dB}$ and $M=2$

Firstly, we analyze the clustering effect of FCM clustering algorithm under this feature. Set the simulation parameters: cooperative SU $M=2$, sampling points $N=1000, S N R=-11 \mathrm{~dB}$. Figure 4 shows the signal and noise feature training samples obtained by the feature extraction method of split recombination combined with information geometry as the training input of the classifier.

Figure 5 shows the clustering data after using the fuzzy c-means clustering algorithm. The blue dot in Fig. 5 represents the noise feature vector, and the red dot represents the signal feature vector. Black dots and black triangles

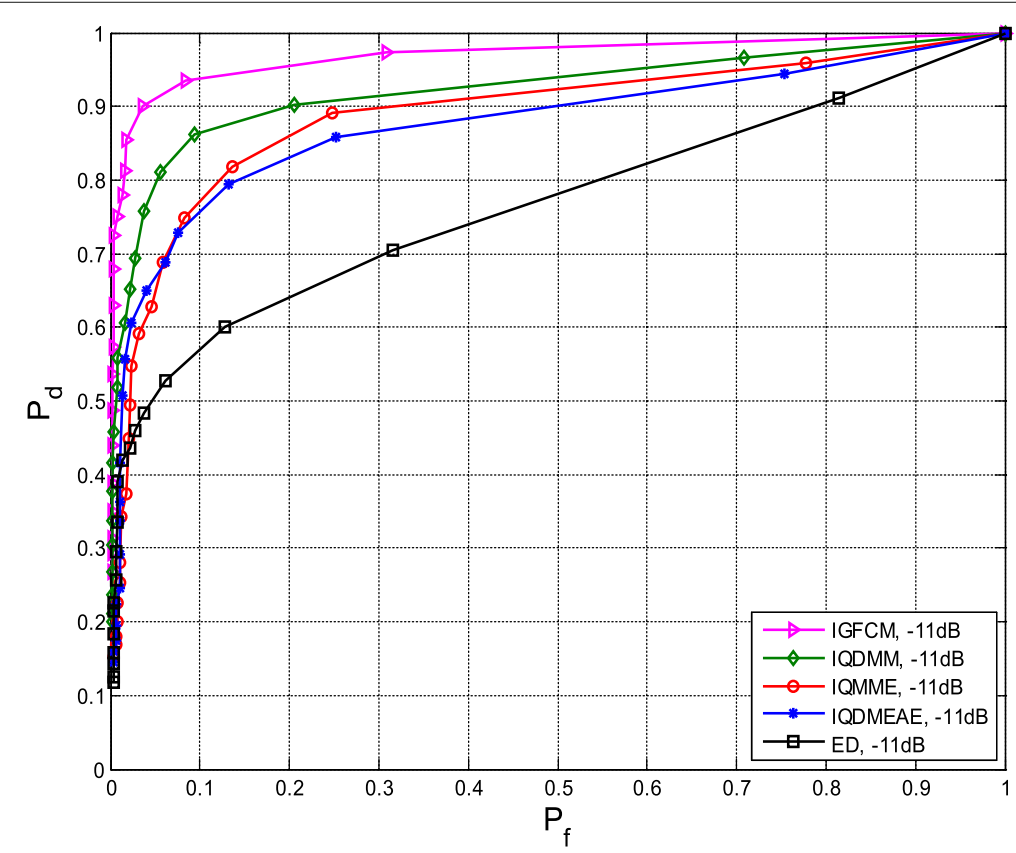

Fig. $7 \mathrm{ROC}$ curves for different algorithms at $S N R=-11 \mathrm{~dB}$ and $M=2$ 


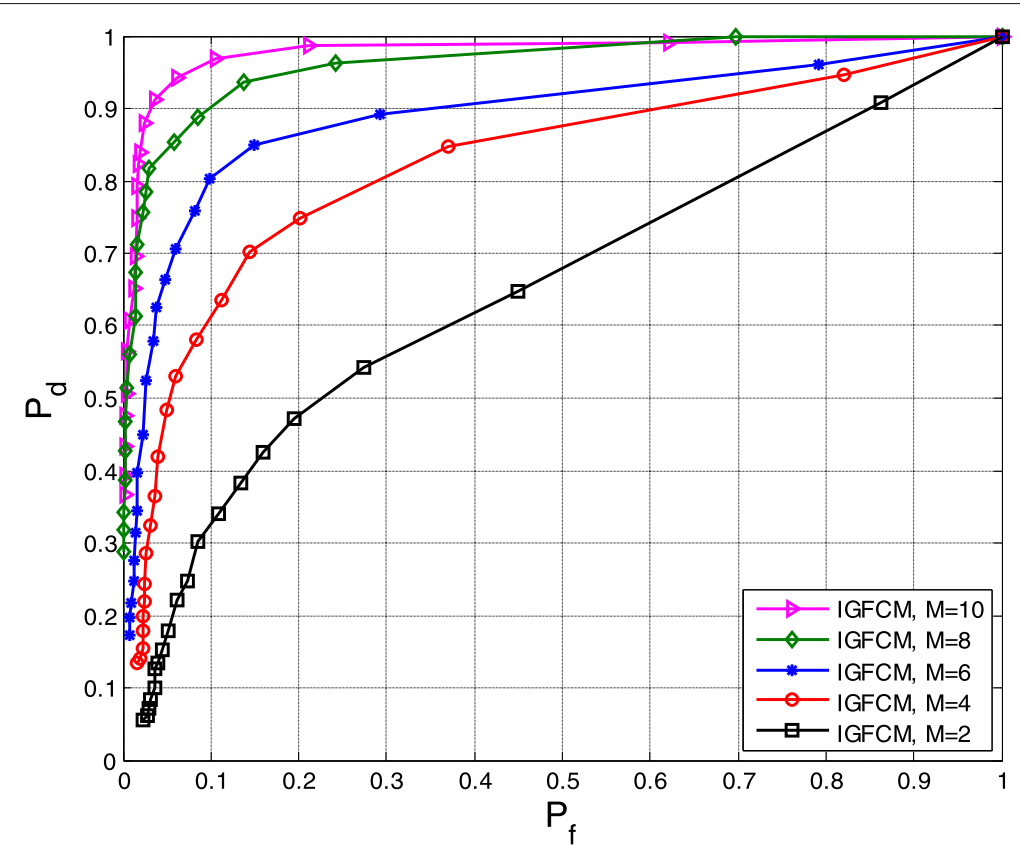

Fig. 8 ROC curve at different number of $S U$ with $S N R=-15 \mathrm{~dB}$

represent the center of the noise class and the PU signal class, respectively.

Further, we compare and analyze the performance of IGFCM and other methods, which are respectively characterized by energy (ED), and the IQMME, IQDMM, IQDMEAE methods are proposed in literature [22]. Given the simulation parameters, the cooperative SU number
$M=2$, the number of sampling points $N=1000$, and the simulation diagrams when the $S N R=-13 \mathrm{~dB}$ and $S N R=-11 \mathrm{~dB}$ respectively are shown in Figs. 6 and 7 . Compared with other methods, the IGFCM has better spectrum sensing performance.

Given the number of sampling points $N=1000$, the $S N R=-15 \mathrm{~dB}$. The simulation results obtained when

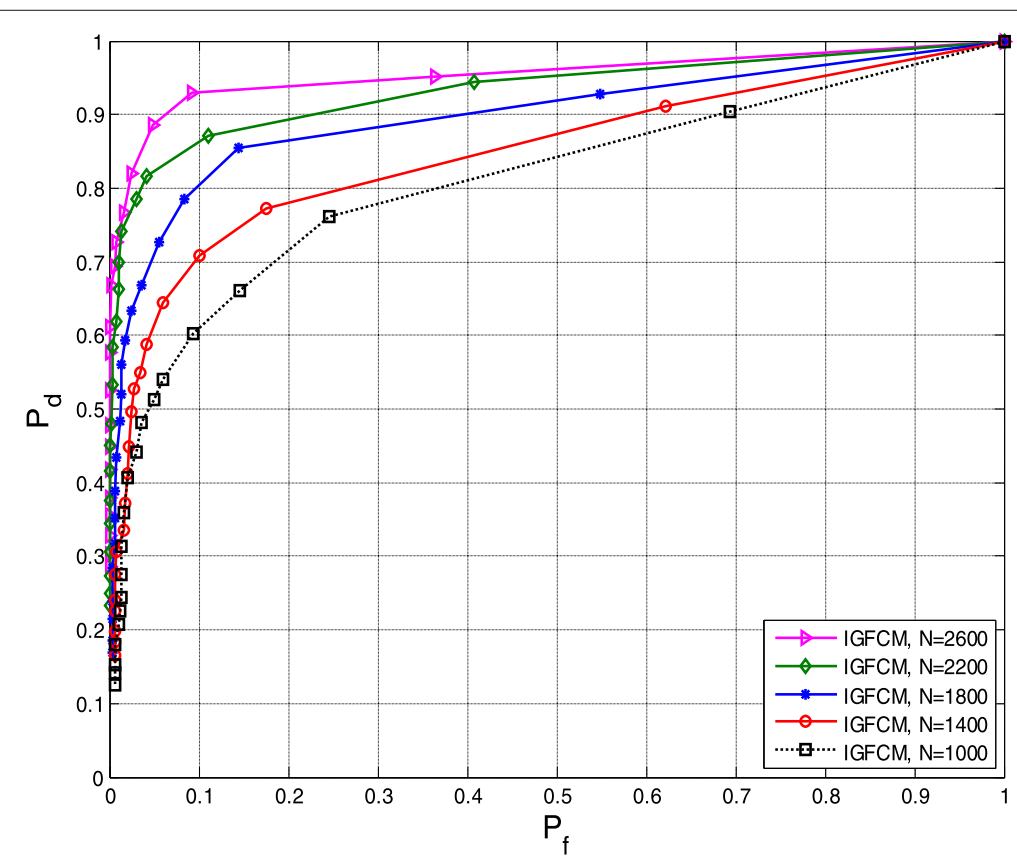

Fig. 9 ROC curve at different sampling points with $S N R=-13 \mathrm{~dB}$ 
the number of SUs is $2,4,6,8$, and 10 respectively are as shown in Fig. 8. As can be seen from Fig. 8, the number of cooperative SUs $M$ has a great relationship with the detection probability of the algorithm. As $M$ increases, the detection probabilities of several algorithms increase to varying degrees.

As the number of sampling points increases, the perceived signal information is more comprehensive, so the extracted features are more representative. In order to observe the spectral sensing performance of the IGFCM method under different sampling points, keep the simulation parameters $M=2$ and $S N R=-13 \mathrm{~dB}$ unchanged. The IGFCM algorithm simulation diagram obtained when the number of sampling points is $1000,1400,1800,2200$, or 2600 respectively. As can be seen from Fig. 9, the spectrum sensing performance increases as the number of sampling points increases.

\section{Conclusion}

In this paper, a spectrum sensing method based on information geometry and FCM clustering is proposed. In the feature extraction process, a feature extraction method combining split recombination and information geometry is proposed to transform complex signal detection problems into manifolds. Geometric problems on the indirect analysis of signal detection problems using geometric tools. Finally, the FCM clustering algorithm is used to train the extracted features to obtain a classifier for spectrum sensing to realize spectrum sensing. The perceptual performance of the method described in this paper is further analyzed in the experimental part. The experimental results show that the method improves the spectrum sensing performance to some extent. In the future work, we will continue to study the application of clustering algorithms in spectrum sensing, such as kernel fuzzy cmeans clustering (KFCM) and the scope of possibilistic fuzzy c-means (PFCM). It is hoped that the spectrum sensing performance can be further effectively improved.

\begin{abstract}
Abbreviations
AWGN: Additive white Gaussian noise; CFAR: Constant false alarm rata; CR: Cognitive radio; DAR: Decomposition and recombination; DMEAE: Difference between the maximum eigenvalue and the average energy; DMM: Difference between the maximum eigenvalue and the minimum feature; FCM: Fuzzy c-means; GMM: Gaussian mixture model; I-DAR: Interval decomposition and recombination; IGFCM: A cooperative spectrum sensing method based on information geometry and fuzzy c-means clustering algorithm; KFCM: Kernel fuzzy c-means; MME: Maximum to minimum eigenvalue; NN: Neural network; O-DAR: Order decomposition and recombination; PFCM: The scope of possibilistic fuzzy c-means; PU: Primary user; SNR: Signal-to-noise ratio; SPD: Symmetric positive definite; SU: Secondary user; SVM: Support vector machine
\end{abstract}

\section{Acknowledgements}

The author wants to thank the author's organization because they have provided us with many conveniences.

\section{Funding}

This work was supported in part by special funds from the central finance to support the development of local universities under No. 400170044, the project supported by the State Key Laboratory of Management and Control for Complex Systems, Institute of Automation, Chinese Academy of Sciences under grant No.20180106, the degree and graduate education reform project of Guangdong Province under grant No.2016JGXM_MS_26, the foundation of key laboratory of machine intelligence and advanced computing of the Ministry of Education under grant No.MSC-201706A and the higher education quality projects of Guangdong Province and Guangdong University of Technology.

\section{Availability of data and materials}

The materials are mainly from different journals and conferences, as shown in the references. In the simulation results and performance analysis section, mainly use MATLAB tools to simulate.

\section{Authors' contributions}

SZ provided this idea and wrote the manuscript. YW demonstrated the idea. JL and $Y Z$ designed the experiment and conducted the experimental verification. PW and NL give constructive suggestions for the structure of the paper. All authors read and approved the final manuscript.

\section{Authors' information}

Shunchao Zhang received his B.S. degree in Hunan Institute of Engineering in 2016. He is currently pursuing the M.S. degree in Guangdong University of Technology, Guangdong, China. His research interests include spectrum sensing in cognitive radio.

Yonghua Wang received his B.S. degree in Electrical Engineering and Automation from Hebei University of Technology in 2001, the M.S. degree in Control Theory and Control Engineering from Guangdong University of Technology in 2006, and Ph.D. degree in Communication and Information System from SUN-YAN SEN University in 2009. He now with School of Automation in Guangdong University of Technology.

Jiangfan Li received his B.S. degree in Foshan University in 2016. He is currently pursuing the M.S. degree in Guangdong University of Technology,

Guangdong, China. His research interests include spectrum sensing in cognitive radio.

Pin Wan received his B.S. degree in Electronic Engineering from Southeast University in 1984, the M.S. degree in Circuit and System from Southeast University in 1990, and Ph.D. degree in Control Theory and Control Engineering from Guangdong University of Technology in 2011. He currently is a professor in School of Automation in Guangdong University of Technology. Yongwei Zhang received his B.S. degree in Jiaying University in 2016. He is currently pursuing the M.S. degree in Guangdong University of Technology, Guangdong, China. His research interests include spectrum sensing in cognitive radio.

Nan Li is currently pursuing the B.S. degree in Guangdong University of Technology, Guangdong, China. Her research interests include spectrum sensing in cognitive radio.

\section{Competing interests}

The authors declare that they have no competing interests.

\section{Publisher's Note}

Springer Nature remains neutral with regard to jurisdictional claims in published maps and institutional affiliations.

\section{Author details}

${ }^{1}$ School of Automation, Guangdong University of Technology, Guangzhou 510006, China. ${ }^{2}$ State Key Laboratory of Management and Control for Complex Systems, Institute of Automation, Chinese Academy of Sciences, Beijing 100190, China. ${ }^{3}$ Hubei Key Laboratory of Intelligent Wireless Communications, South-Central University for Nationalities, Wuhan 430074, China.

Received: 26 August 2018 Accepted: 4 January 2019

Published online: 23 January 2019

\section{References}

1. A. A. Khan, M. H. Rehmani, M. Reisslein, Cognitive radio for smart grids: survey of architectures, spectrum sensing mechanisms, and networking protocols. IEEE Commun. Surv. Tutor. 18, 860-898 (2016)

2. L. Xiao, in IEEE Global Communications Conference: 2014; Austin. Prospect theoretic analysis of anti-jamming communications in cognitive radio networks (IEEE, Austin, 1996), pp. 746-751 
3. K. Cichon, A. Kliks, H. Bogucka, Energy-efficient cooperative spectrum sensing: a survey. IEEE Commun. Surv. Tutor. 18, 1861-1886 (2016)

4. N. S. Kim, J. M. Rabaey, A dual-resolution wavelet-based energy detection spectrum sensing for UWB-based cognitive radios. IEEE Trans. Circ. Syst. I Regular Papers. PP, 1-14 (2017)

5. E. Chatziantoniou, B. Allen, V. Velisavljevic, P. Karadimas, J. Coon, Energy detection based spectrum sensing over two-wave with diffuse power fading channels. IEEE Trans. Veh. Technol. 66, 868-874 (2017)

6. X. Zhang, R. Chai, F. Gao, in IEEE Global Conference on Signal and Information Processing:09 February 2015; Atlanta. Matched filter based spectrum sensing and power level detection for cognitive radio network (IEEE, Atlanta, 2015), pp. 1267-1270

7. A. Surampudi, in India International Conference on Information Processing: 2016. An adaptive decision threshold scheme for the matched filter method of spectrum sensing in cognitive radio using artificial neural networks (IEEE, Doha, 2016), pp. 1-5

8. R. Mahapatra, in IEEE International Symposium on Wireless Communication Systems: 2016;Reykjavik. Cyclostationary detection for cognitive radio with multiple receivers (IEEE, Reykjavik, 2008), pp. 493-497

9. W. Zhang, G. Abreu, M. Inamori, Y. Sanada, Spectrum sensing algorithms via finite random matrices. IEEE Trans. Commun. 60, 164-175 (2012)

10. Y. Zeng, Y. C. Liang, Eigenvalue-based spectrum sensing algorithms for cognitive radio. IEEE Trans. Commun. 57, 1784-1793 (2009)

11. C. Liu, in 7th International Conference on Wireless Communications, Networking and Mobile Computing: 2011; Wuhan. A distance-weighed algorithm based on maximum-minimum eigenvalues for cooperative spectrum sensing (IEEE, Wuhan, 2011), pp. 1-4

12. N. Liu, H. S. Shi, B. Yang, D. P. Yuan, Spectrum sensing method based on ME-S-ED. Meas. Control. Technol. 35, 125-128 (2016)

13. M. T. Antonia, V. Sergio, Random matrix theory and wireless communications. Commun. Inf. Theory. 1, 1-182 (2004)

14. W. Hu, in International Conference on Wireless Communications, Networking and Mobile Computing: 26-28 September 2014; Beijing. Cooperative spectrum sensing algorithm based on bistable stochastic resonance (IET, Beijing, 2014), pp. 126-130

15. J. K. Liu, X. S. Wang, W. Tao, Q. U. Long-Hai, Application of information geometry to target detection for pulsed-Doppler radar. J. Natl Univ. Defense Technol. 33, 77-80 (2011)

16. Q. Chen, in International Conference on Cloud Computing and Security: 16-18 June 2017; Nanjing. Research on cognitive radio spectrum sensing method based on information geometry (IEEE, Nanjing, 2017), pp. 554-564

17. Q. Lu, S. Yang, F. Liu, Wideband spectrum sensing based on Riemannian distance for cognitive radio networks. Sensors. 17, 661 (2017)

18. L. Xiao, Y. Li, G. Han, W. Zhuang, PHY-layer spoofing detection with reinforcement learning in wireless networks. IEEE Trans. Veh. Technol. 65 , 10037-10047 (2016)

19. S. P. Maity, S. Chatterjee, T. Acharya, On optimal fuzzy c-means clustering for energy efficient cooperative spectrum sensing in cognitive radio networks. Dig. Signal Proc. 49, 104-115 (2016)

20. A. Paul, S.P. Maity, Kernel fuzzy c-means clustering on energy detection based cooperative spectrum sensing. Dig. Commun. Netw. 4, 196-205 (2016)

21. V. Kumar, in Twenty Second National Conference on Communication: 4-6 March 2016;Guwahati. K-mean clustering based cooperative spectrum sensing in generalized k-u fading channels (IEEE, Guwahati, 2016), pp. 1-5

22. Y. Zhang, P. Wan, S. Zhang, Y. Wang, N. Li, A spectrum sensing method based on signal feature and clustering algorithm in cognitive wireless multimedia sensor networks. Adv. Multimedia. 2017, 1-10 (2017)

23. K. M. Thilina, K. W. Choi, N. Saquib, E. Hossain, Machine learning techniques for cooperative spectrum sensing in cognitive radio networks. IEEE J. Sel. Areas Commun. 31, 2209-2221 (2013)

24. G. C. Sobabe, in International Congress on Image and Signal Processing, Biomedical Engineering and Informatics. A machine learning based spectrum-sensing algorithm using sample covariance matrix (IEEE, Shanghai, 2018), pp. 1-6

25. S. Chatterjee, A. Banerjee, T. Acharya, S. P. Maity, Fuzzy c-means clustering in energy detection for cooperative spectrum sensing in cognitive radio system. Proc. Mult. Access Commun. 8715, 84-95 (2014)

26. A. S. Kannan, in International Conference on Control Communication and Computing: 13-15 December 2013; Thiruvananthapuram. Performance analysis of blind spectrum sensing in cooperative environment (IEEE, Thiruvananthapuram, 2013), pp. 277-280
27. L. Xiao, J. Liu, Q. Li, N. B. Mandayam, H. V. Poor, User-centric view of jamming games in cognitive radio networks. IEEE Trans. Inf. Forensics Secur. 10, 2578-2590 (2015)

28. C. Lenglet, M. Rousson, R. Deriche, O. Faugeras, Statistics on the manifold of multivariate normal distributions: theory and application to diffusion tensor MRI processing. J. Math. Imaging Vis. 25, 423-444 (2006)

29. M. Menendez, A differential geometric approach to the geometric mean of symmetric positive-definite matrices. Siam J. Matrix Anal. Appl. 26, 735-747 (2008)

30. M. Menendez, D. Morales, L. Pardo, M. Salicru, Statistical tests based on geodesic distances. Appl. Math. Lett. 8, 65-69 (1995)

31. M. Calvo, J. M. Oller, A distance between multivariate normal distributions based in an embedding into the Siegel group. J. Multivar. Anal. 35 , 223-242 (1991)

32. M. N. Ahmed, S. M. Yamany, N. Mohamed, A. A. Farag, T. Moriarty, A modified fuzzy c-means algorithm for bias field estimation and segmentation of MRI data. IEEE Trans. Med. Imaging. 21, 193-199 (2002)

33. D. M. S. Bhatti, in International Conference on Information and Communication Technology Convergence: 18-20 October 2017; Jeju. Fuzzy c-means and spatial correlation based clustering for cooperative spectrum sensing (IEEE, Jeju, 2017), pp. 486-491

34. D. M. S. Bhatti, N. Saeed, H. Nam, Fuzzy c-means clustering and energy efficient cluster head selection for cooperative sensor network. Sensors. 16, 1459-1476 (2016)

\section{Submit your manuscript to a SpringerOpen ${ }^{\circ}$ journal and benefit from:}

- Convenient online submission

- Rigorous peer review

- Open access: articles freely available online

- High visibility within the field

- Retaining the copyright to your article

Submit your next manuscript at $>$ springeropen.com 